\title{
Synthesis and Nonlinear Optical Properties of Novel T-Type Polyimide with Exceptional High Thermal Stability of Second Harmonic Generation
}

\author{
Ju-Yeon Lee, ${ }^{*}$ Han-Bae Bang, Chang Suk Baek, Eun-Ju Park, Bum Ku Rhee, ${ }^{\dagger}$ and Seung Mook Lee ${ }^{\dagger}$ \\ Institute for Nanotechnologv Applications, Department of Chemistry, Inje Chivensitv, Kimhae 621-749. Korea \\ ${ }^{\dagger}$ Deparment of Physics, Sogang Chiversity. Seoul 121-7+2, Korea \\ Received Januarv 12. 2004
}

Key Words : Nonlinear optics (NLO). Polyimides. Differential scanning calorimetry (DSC). Atomic force microscopy (AFM). SHG coefficient

Extensive studies have been made for the synthesis of nonlinear optical (NLO) polymers over the past decade because of their potential applications in the field of telecommunications. optical signal processing and optical switching. One of the current challenges is to design novel NLO polymers having optimized properties. In the developments of NLO polymers for electro-optic device applications. stabilization of electrically-induced dipole alignment is an important consideration. Two approaches to minimize the randomization have been proposed. One is to use crosslinking method ${ }^{1-4}$ and the other is to use high $T_{8}$ polymers such as polyimides ${ }^{5-7}$ Poly imide materials for NLO applications have attracted attentions because of their high glass transition temperature $\left(T_{z}\right)$. which can be utilized to stabilize the dipole orientation of the NLO chromophore at high temperatures. Another attractive merit of poly imides is their high thermal stability that enables them to endure at elevated temperature during operation in electro-optic devices. There are two types of NLO polyimides. which have been used either as polymer hosts for composite materials or as polymer backbones for side-chain NLO chromophores. Side-chain polymer systems have the advantages such as homogeneity and high level of NLO chromophore relative to the hostguest systems. which suffer from poor homogeneity of chromophores and diffusion of the chromophores at high temperatures. In this work we prepared novel T-type polyimides containing 2.5-dioxynitrostilbenyl groups as NLO-chromophores. We selected 2.5-dioxynitrostilbenyl group as a NLOchromophore because it has a large dipole moment and is rather easy' to synthesize. Furthermore 2.5-dioxynitrostilbeny'1 group constitutes a novel T-type NLO polyimides. and these T-type NLO polyimides are not presented in the literature. Thus. we designed and syinthesized a new type of NLO polyimide. in which the pendant NLO chromophores are parts of the polymer backbones. These mid-type NLO polymers are expected to have both of the merits of main chain- and side chain-NLO polymers: stabilization of dipole alignment and good solubility. After confirming the structure of the resulting polymers we investigated the properties such as solubility $T_{\mathrm{g}}$. thermal stability surface morphology of polymer film. second harmonic generation (SHG) activity

\footnotetext{
Corresponding Author. Phone: +82-55-320-3221; Fax: +82-55-
} 32 1-9718; e-mail: chemliyiàinje.ackr and relaxation of dipole alignment. We now report the results of the initial phase of the work.

2.5-Di-(2'-hydroxyethoxy)-4'-nitrostilbene (2) was prepared according to a literature procedure. ${ }^{8}$ Chromophorecontaining dianhydride 3 was prepared by the reaction of diol 2 with trimellitic anhydride acid chloride in dry NMP in the presence of pyridine. The polyimide 4 was synthesized by reacting aromatic dianlydride monomer 3 with stoichiometric amounts of 4.4'-(hexafluoroisopropylidene)dianiline in $m$-cresol. Synthetic method of polymer 4 is summarized in Scheme 1. The resulting polymer was purified by Soxhlet extraction for 2 days with diethyl ether as a solvent. The striking feature of this polymer is that it has pendant NLO chromophores that are parts of the polymer main chains. Thus. we obtained a new type of NLO polyimide with side chain and main chain characteristics simultaneously. These mid-type NLO polymers are expected to have both of the merits of main chain and side chain NLO polymers. The chemical structures of the resulting polymers were confirmed by ${ }^{1} \mathrm{H}$ NMR. IR spectra and elemental analy'sis. ${ }^{1} \mathrm{H} N \mathrm{NR}$ spectra of the polymers showed a signal broadening due to polymerization. but the chemical shifts are consistent with the proposed polymer stnuctures. The IR spectra supported the formation of poly imide. The IR spectrum of the polymer sample shows peaks near 1780 and $725 \mathrm{~cm}^{-1}$ that are characteristic bands of imide asymmetric carbonyl stretching and imide ring deformation, respectively. IR spectrum of the same polymer sample also shows strong absorption peaks near $1728 \mathrm{~cm}^{-1}$ and $1374 \mathrm{~cm}^{-1}$ due to a symmetric carbonyl stretching and $\mathrm{C}-\mathrm{N}$ stretching of imide ring. respectively: These results are consistent with the proposed structures. indicating that the NLO-chromophores remained intact during the polymerization. We now have well defined polymer 4 and investigate their properties.

The number average molecular weight $\left(M_{\mathrm{n}}\right)$ determined by gel permeation cluromatography' (GPC) was $23100\left(M_{\mathrm{w}} / M_{\mathrm{n}}=\right.$ $1.64)$ for polymer 4 . The polyimide 4 was soluble in common solvents such as acetone. DMF, and DMSO, but was not soluble in methanol and diethyl ether. The thermal behavior of the polymers were investigated by thermogravimetric analy sis (TGA) and differential scauning calorimetry (DSC) to determine the thermal degradation pattern and glass transition temperature $\left(T_{\mathrm{g}}\right)$. Polymer 4 showed a thermal stability up to $360^{\circ} \mathrm{C}$ from their TGA thermograms. The 


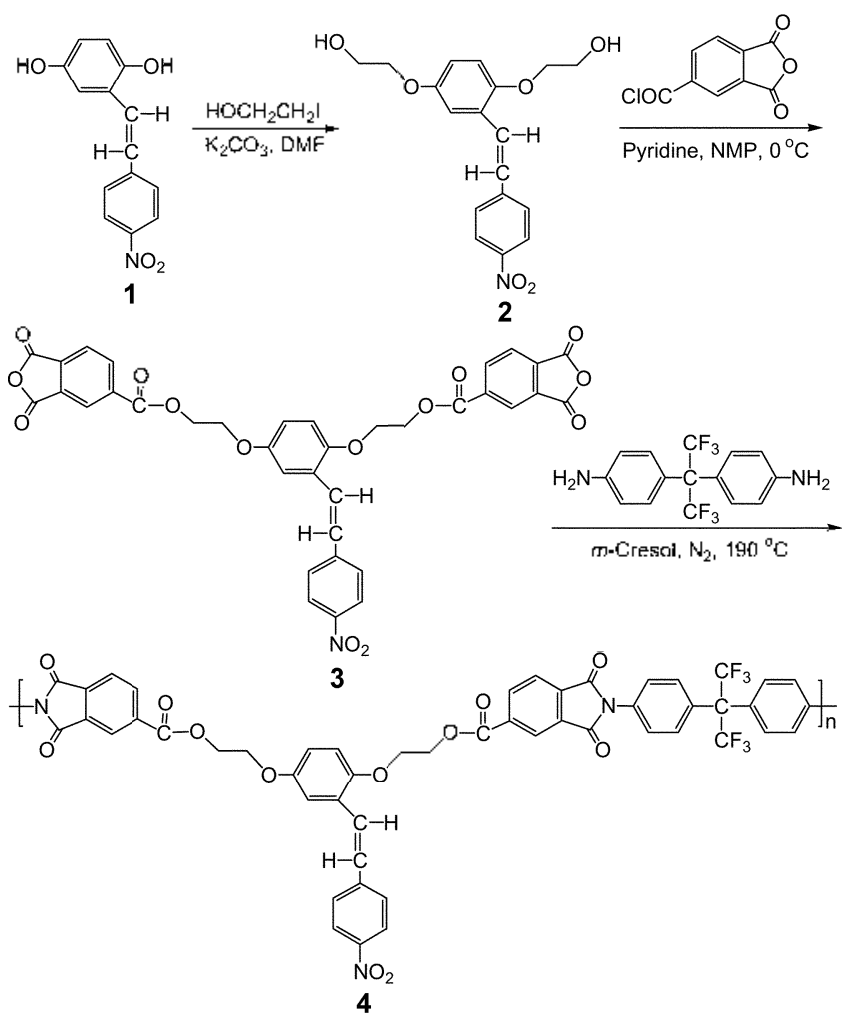

Scheme 1

initial weight loss in the polymers begins at $360^{\circ} \mathrm{C} . \tau_{g}$ value of the polymer 4 measured by DSC were around $153^{\circ} \mathrm{C}$. This is a rather low $T_{z}$ value compared to those of common rigid polyimides and probably attributed to the flexibility in the polymer backbone containing ether linkages.

The NLO properties of polymers were studied by the $\mathrm{SHG}$ method. To induce noncentrosymmetric polar order, the spin-coated polymer films were corona-poled. As the temperature was raised to 170 " $\mathrm{C}, 6 \mathrm{kV}$ of corona voltage was applied and kept $170^{\circ} \mathrm{C}$ for $30 \mathrm{~min}$. After the electric poling. the dipole moments of the NLO-chromophores were aligned and UV-Vis spectrum of polymer 7 exhibited a slight blue shift and a decrease in absorption due to birefringence. The estimated order parameter value $\boldsymbol{D}$ was found to have a value of 0.16 for polymer $4\left(\Phi^{-}-1-A_{1} / A_{0}, A_{0}\right.$ and $A_{1}$ are the absorbances of the polymer film before and after poling). The decrease in absorbance after poling is an indicator of the dipole alignment. The refractive index of the sample was measured by the optical transmission technique. ${ }^{9} \mathrm{SHG}$ measurements were performed at a fundamental wavelength of $1064 \mathrm{~nm}$ using a mode locked Nd-YAG laser. ${ }^{10.11}$ In order to determine the microscopic second-order susceptibility of the polymer, the angular SHG dependence was recorded. The values of $d_{3}$ and $d_{3 ;}$ for polymer 4 were $1.29 \times 10^{-9}$ esu $(0.54 \mathrm{pm} / \mathrm{V})$ and $3.48 \times 10^{-9} \mathrm{esu}(1.46 \mathrm{pm} / \mathrm{V})$, respectively. Since the second harmonic wavelength was at $532 \mathrm{~nm}$, which is not in the absorptive region of the resulting polymer, there was not resonant contribution to this $d_{3,3}$ value. To evaluate the high-temperature stability of the polymers, we studied the temporal stability of the $\mathrm{SHG}$ signal. In Figure 1, we present the dynamic thermal stability

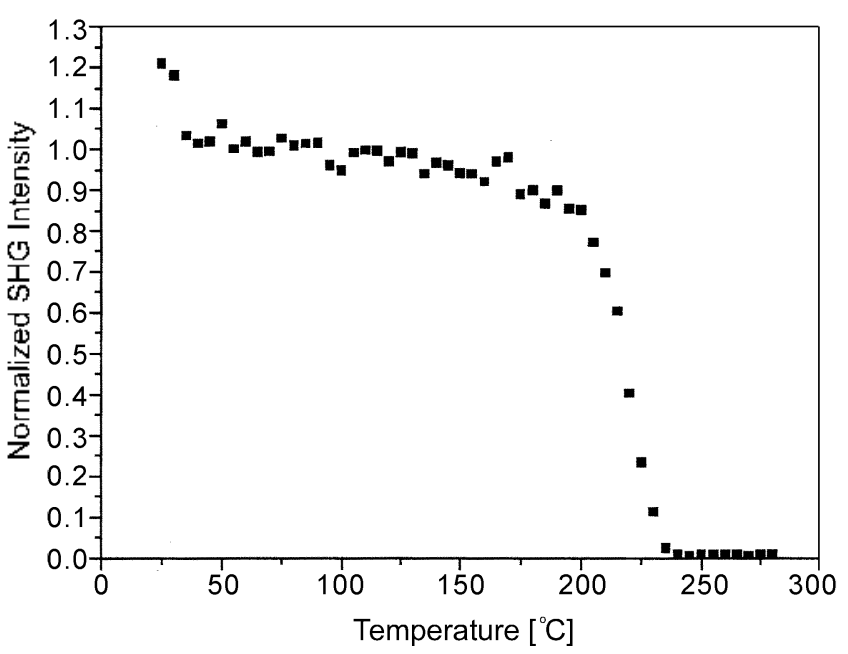

Figure 1. Normalized $d_{3}$ of polymer 4 as a tunction of temperature at a hoating rate of $10^{\circ} \mathrm{C} / \mathrm{m}$ min.

study of the NLO activity of the film 4. To investigate the real time NLO decay of the $\mathrm{SHG}$ signal of the poled polyıner films as a function of temperature, in situ SHG measurements were performed at a heating rate of $10^{\circ} \mathrm{C} / \mathrm{min}$ from 30 ${ }^{\circ} \mathrm{C}$ to $300^{\circ} \mathrm{C}$. The polymer film exhibited a thermal stability even at $45^{\circ} \mathrm{C}$ higher than $T_{\mathrm{k}}$ and no significant $\mathrm{SHG}$ decay was observed below $200{ }^{\circ} \mathrm{C}$. In general, side chain $\mathrm{NLO}$ polyıners loose thermal stability of dipole alignınent around $T$. Stabilization of dipole alignment is a characteristic of main chain NLO polymers. The exceptional high thermal stability of second harmonic generation of polymer 4 is due to the stabilization of dipole alignment of NLO chromophore, which stems from the partial main chain character of the polymer structure. Thus, we obtained a new type of NLO polyimide having both of the merits of main chain- and sidechain NLO polymers; stabilization of dipole alignment and good solubility. We are now in the process of extending the polymerization system to the synthesis of other type of NLO polymers and the results will be reported elsewhere.

Acknowledgement. This work was supported by Korea Research Foundation Grant (KRF-2003-041-D20173).

\section{References}

1. Ilan, K. S.: Park, S. K.: Shim, S. Y.: Jahng. W. S.: Kim, N. J. Bull. Korean Chem Soc 1998. 19. 1165.

2. Han. K. S.: l'ark. S. K.: Sliim. S. Y.: Lee. Y. S.: Jahng. W. S.: Kim. N. J. Bull Koreun ( hem. Soc. 1998. 19. 1168.

3. Lee. J.-H.: Loe. K.-S. Bull. Foreun (hem. Sor 2000. 21. 847.

4. Cho, B. R.: Kim, Y. II,: Son, K, W.: Khalil, C.: Kim, Y. II.: Jeon, S.-1. Bull . Norean Chent. Soc. 2002. 23. 1253.

5. Yu. D.: (Sharavi. A.: Yu. J.. J. An. Chent Soc 1995. 117. 11680.

6. Chen. L. Jen. A. K. Y.: Cai. Y. J. 1m. (hem Soc. 1995. /I7. 7295.

7. Km. M. H.: Jin. J.-I.: Lee. C. J.: Kinl. N. J.: Paarh. C. H. Bull. Aorean Chem. Sor 2002. 23. 964.

8. I,ee. J.-Y.: 1.ee, W.-I.: Park, F.,-I.: Bang, II,-B.: Rhee. B. K.: Jung

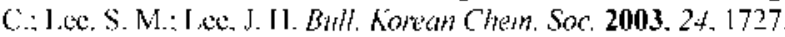

9. Cisneres. J. I. Appt Opt 1998. 37. 5262.

10. Maker: P. L.: lerhune. R. W.: Nisenolf: M.: Savage. C. M. Ph: Rev. Lett. 192.8.21.

11. Herman. W. N.: Hayden. L. M. J. Opt Soc Am. B 1995. /2. 416 . 